\title{
Sparse-Based Classification with Sub-dictionary Division and Weighted Combination
}

\author{
Junyan Wang ${ }^{1, a}$, Chunmei Zhang ${ }^{2, b}$, Dan $\mathrm{Li}^{3, \mathrm{c}}$ \\ ${ }^{1}$ Department of Computer Science and Engineering, Beifang University of Nationalities, Yinchuan, \\ 750021, China \\ ${ }^{2}$ Department of Computer Science and Engineering, Beifang University of Nationalities, Yinchuan, \\ 750021, China \\ ${ }^{3}$ Department of Computer Science and Engineering, Beifang University of Nationalities, Yinchuan, \\ 750021, China \\ aemail: 513587346@qq.com, bemail: chunmei66@hotmail.com \\ cemail: 471296247@qq.com
}

Keywords: Sparse Representation; Classification; Sub-dictionary Division; Weighted Combination

\begin{abstract}
A novel classification approach for hyperspectral data based on sparse representation framework is proposed in this paper. This new method divides all spectrums of hyperspectral samples to sub-dictionaries based on spectral characteristics of the data themselves. Meanwhile, a weighting algorithm based on minimum residual is adopted to combine sub-dictionaries. Experimental results show that our proposal obtained higher accuracy comparing with that of a whole dictionary.
\end{abstract}

\section{Introduction}

Sparse representation is a new emerging theory after wavelets in signal processing community [1][2]. It was rapidly interested in 2000's and developed in image applications successfully [3] [4] [5] [6]. In the decade of 2010s, sparse representation has gained considerable attention by the scholars around the world and employed in many research fields recently [7] [8].

Pixel classification is one of the most important parts in the research of hyperspectral remote sensing. Sparse decomposition algorithm is an effective framework and can be implemented to classify high dimensional data. The framework has the advantages that it does not need dimension reduction and model selection, as well as dictionary training. Each test data can be sparsely represented with only a few non-zero decomposition coefficients by a few atoms of an overcomplete dictionary which are constructed by training samples directly. According to the reconstruction error, test data can be classified to the corresponding class. Several researchers have improved the classification accuracy of remote sensing data based on sparse representation framework [9] [10].

SR (sparse representation) algorithm can use the training set directly to form the over-complete dictionary for speeding and simplifying classification algorithm. However, simply putting the training samples of the same category with all of their components together to form a dictionary for hyperspectral classification is not a good idea because of the much higher dimension of such data. It couldn't capture the intrinsic spectral characteristics of the test data more precisely especially in a noise environment. In order to improve the classification accuracy, we proposed a method of dividing spectrum to sub-dictionaries based on the spectral characteristics of the data themselves, and then use a weighting algorithm to combine the sub-dictionaries in which the weight of each sub-dictionary is based on their minimum residual.

This paper is organized as follows. In section 2, introductions on sparse representation and its algorithm are given briefly at 2.1 and 2.2. Then our method is presented in detail at 2.3. Experiments and results on Botswana data of NASA EO-1 satellite which is compared with SR algorithm are showed in section 3. Finally, conclusions of the paper are revealed in section 4 . 


\section{Methodology}

\subsection{Sparse Representation}

Sparse representation can be addressed as follows: Let, $y \in R^{n}$ is an input pattern which can be represented approximately as:

$$
y \approx D \alpha
$$

where, $D \in R^{n \times m}$ is an over-completed dictionary of atoms $\left\{d_{i}\right\}(i=1,2, \ldots, m), \alpha \in R^{m}$ is a sparse vector with a few non-zero coefficients. The formula can be characterized as:

$$
\underset{\alpha}{\operatorname{argmin}}\|y-D \alpha\| \quad \text { subject to }\|\alpha\|_{0} \leq \Gamma
$$

in which \|\|$_{0}$ is the zero norm and $\Gamma$ is a constraint which limits the number of non-zero coefficients of sparse vector $\alpha$.

A set of dictionaries can be constructed according to different class $c(c=1,2 \ldots k)$. Each dictionary gets the labelled samples of the same class from hyperspectral data to form the overcomplete dictionary. Let $D_{c}=\left[d_{1}, d_{2}, \ldots, d_{m}\right]\left(D \in R^{n \times m}\right)$ denotes the dictionary of $c$-th class.

According to the theory of sparse representation, each test data can be sparsely represented by a few training samples of an over-complete dictionary with only a few non-zero decomposition coefficients. We can get sparse decomposition coefficient according to formula (3).

$$
\alpha_{c}(y)=\arg \min _{\alpha_{c}}\left\|y-D_{c} \alpha_{c}\right\|_{2}^{2}+\lambda\left\|\alpha_{c}\right\|_{1}
$$

The above formula is an $\ell_{1}$-regularized least squares problem. Where $\alpha_{c}=\left[\alpha_{c 1}, \alpha_{c 2}, \ldots \alpha_{c m}\right]^{T}$ is the coefficient of sparse representation of $y$ corresponding to the $c$-th dictionary $D_{c}$.

Regularization parameter $\lambda$ is used to balance the reconstruction error and sparsity. The threshold of parameter $\lambda$ is selected according to empirical value. Then the test data $\mathrm{y}$ is categorized to the corresponding class according to formula (4).

$$
\min _{c} R_{c}(y)=\min _{c}\left(\left\|y-D_{c} \alpha_{c}\right\|_{2}\right)
$$

where $R_{c}(y)$ is the residual to reconstruct $y$ by training samples in the category $c$.

\subsection{Implementation of SR Algorithm}

\section{Algorithm I \\ Implementation of SR Algorithm}

\section{Input: \\ $X$ : Training set (labelled example set) \\ $Y$ : Test set (Unlabelled test data set) \\ Output:}

$c(y)$ : The category of each test data $y$.

Step 1 Each training sample $x \in X$ or test data $y \in Y$ is normalized.

Step 2Use the training set of different categories to form the over-complete dictionaries $D_{c}$ respectively.

Step 3 Use lasso algorithm to decompose the test data sparsely to get the sparse representation coefficient $\alpha_{c}$.

Step 4 According to formula (4), calculate the residual error $R_{c}(y)$ of test data which was sparse approximation by $D_{c}$. Then the test data is categorized to the corresponding class according to the minimum reconstruction error.

Step 5 Stop repeat step 3 and step 4 until all the test data are categorized.

Thanks for the good performance of sparse representation, we could use the training set directly to form the over-complete dictionary for classification applications. However, for hyperspectral 
data with high dimension, if the data were separated according to their spectral characteristics to form sub-dictionaries, we could depict the intrinsic structures of such data precisely. That means classification will be more accurate while the computation becomes a bit complicated.

We propose a method by dividing spectrum to sub-dictionaries following the spectral characteristics of the data themselves, then, assign diffident weights to diffident sub-dictionaries according to their residuals. With this method, we can get a new dictionary in which each subdictionary has its own weight.

\subsection{SR Algorithm with Sub-dictionary Division and Weighted Combination}

The sparse representation classification method for hyperspectral images with sub-dictionary division is implemented in two steps: firstly, calculate the mean spectra curve of training samples of $\mathrm{k}$ categories on all bands. Secondly, all bands are divided based on band number of peaks and troughs of mean spectra curve of $k$ categories. Here, band division is equal to sub-dictionary division.

In our improved SR algorithm based on sub-dictionary division and weighted combination, weight is used to describe the contribution of each sub-dictionary to the classification of test data. Average weight is gotten rid of because of its inability. So we need a new way to make more sense on calculating the weight of each sub-dictionary to the final sparse reconstruction. The new dictionary in matrix form of our improved SR algorithm is listed in formula (5).

$$
D_{c}=\left[w_{1} D_{c}^{1}, w_{2} D_{c}^{2}, \ldots, w_{j} D_{c}^{j}, \ldots, w_{L} D_{c}^{L}\right]
$$

where $j=1,2, \ldots, L$ indicates the index of band division. $D_{c}^{j}$ is the $j$-th sub-dictionary of $c$-th category.

In SR algorithm, the minimum reconstruction error is used to classify the test data. So in the subdictionary division model, minimum reconstruction error of each sub-dictionary reflects the contribution of the specific sub-dictionary to the final classification [11]. So calculation of $W_{j}$ is based on $\min R_{c}^{j}\left(y^{j}\right)$.

The minimum reconstruction error of each sub-dictionary is calculated and normalized respectively, and then the weight of each band region is obtained as follows:

$$
\begin{aligned}
& \min _{c} R_{c}^{j}\left(y^{j}\right)=\min _{c}\left(\left\|y^{j}-D_{c}^{j} \alpha_{c}^{j}\right\|_{2}\right) \\
& w_{j}=\frac{1}{\frac{\min R_{c}^{j}\left(y^{j}\right)}{\min R_{c}^{1}\left(y^{1}\right)}+\frac{\min R_{c}^{j}\left(y^{j}\right)}{\min R_{c}^{2}\left(y^{2}\right)}+\ldots+\frac{\min R_{c}^{j}\left(y^{j}\right)}{\min R_{c}^{L}\left(y^{L}\right)}, j=1,2,3, \ldots, L .} \\
& w_{j}=\frac{1}{\sum_{i=1}^{L} \frac{\min R_{c}^{j}\left(y^{j}\right)}{\min R_{c}^{i}\left(y^{i}\right)}}, i=1,2,3, \ldots, L . j=1,2,3, \ldots, L . \sum_{j=1}^{L} w_{j}=1
\end{aligned}
$$

where, $y^{j}$ is the given band of test data corresponding to sub-dictionary $D_{c}^{j}$. 


\section{Algorithm II \\ Algorithm of Our Method}

\section{Input:}

The matrix of training samples $x=\left(X_{1}, X_{2}, \ldots, X_{k}\right) \in R^{n \times m}$, which contain $k$ classes. Test data $y \in R^{n}$ contained a reconstruction error $\varepsilon>0$.

\section{Output:}

$c(y)$ : the category of test data.

Step 1 Normalize the training samples $X$ to make it $\ell_{2}$-norm.

Step 2 Use the training set to form the over-complete dictionary $D_{c}$.

Step 3 For $c=1, \ldots, k$, calculate mean spectrum curve of $c$-th category and then divide all bands of $D_{c}$ into $L$ sub-dictionaries based on band number of peaks and troughs of mean spectra curve.

Step 4 For $j=1, \ldots, L$, use lasso algorithm to decompose the test data $y^{j}$ sparsely to get the minimum reconstruction error $R_{c}^{j}\left(y^{j}\right)$.

Step 5 According to formula (7), calculate weight of each sub-dictionary. Then the test data is classified to the corresponding class according to minimum accumulative residual error.

Step 6 Stop repeat step 4 and step 5 until all the test data are categorized.

Figure 1 presents the block diagram of the proposed method.

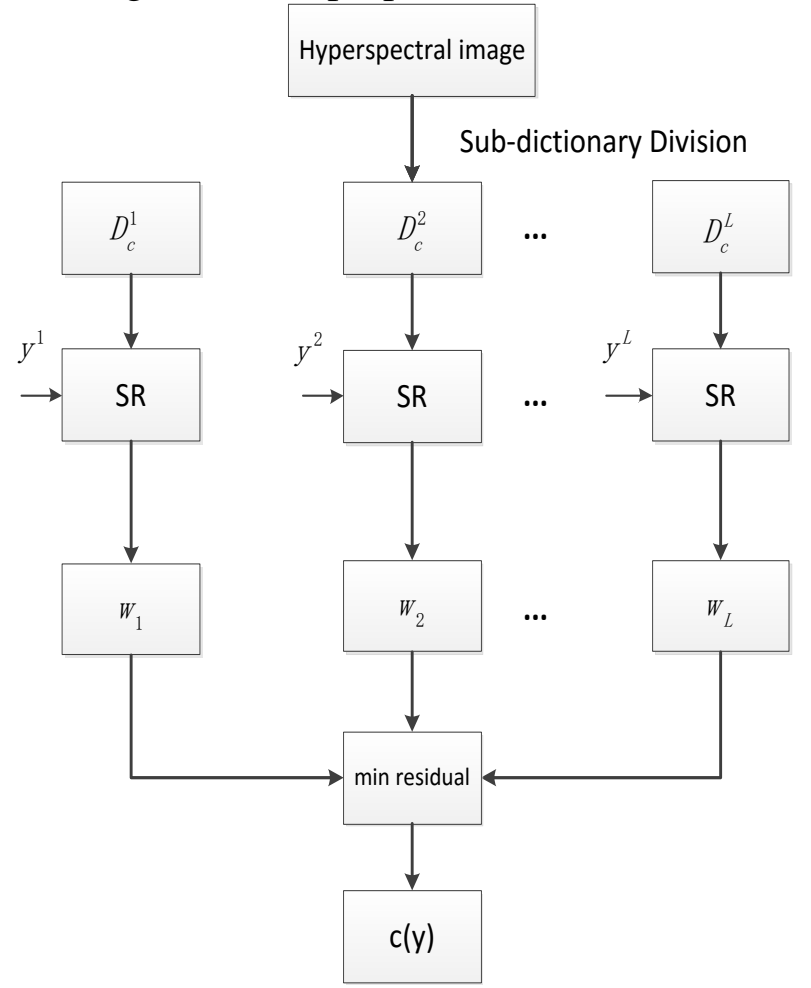

Fig. 1 The block diagram of the proposed method, $y^{j}$ is the given band of test data corresponding to sub-dictionary $D_{c}^{j}$.

\section{Experiment}

\subsection{Sub-dictionary Division}

The NASA EO-1 satellite acquired a sequence of data over the Okavango Delta, Botswana in 2001-2004. The Hyperion sensor on EO-1 acquires data at $30 \mathrm{~m}$ pixel resolution over a $7.7 \mathrm{~km}$ strip in 242 bands covering the $400-2500 \mathrm{~nm}$ portion of the spectrum in $10 \mathrm{~nm}$ windows. Uncalibrated and noisy bands that cover water absorption features were removed, and the remaining 145 bands were included as candidate features. The data contains 14 typical ground objects and 145 bands. 
Calculating mean spectrum curve of 14 categories on 145 bands, we can show the results in figure 2 .

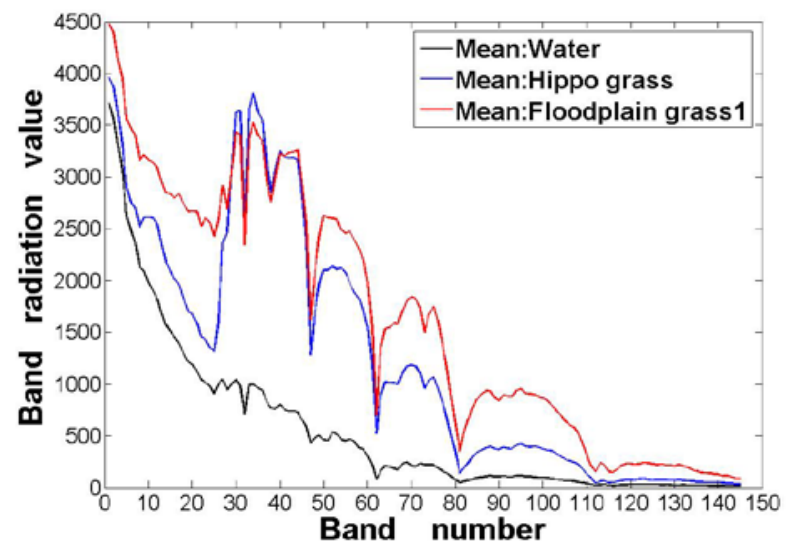

(a)Mean spectrum curve of $1^{\text {st }}$ to $3^{\text {th }}$ category.

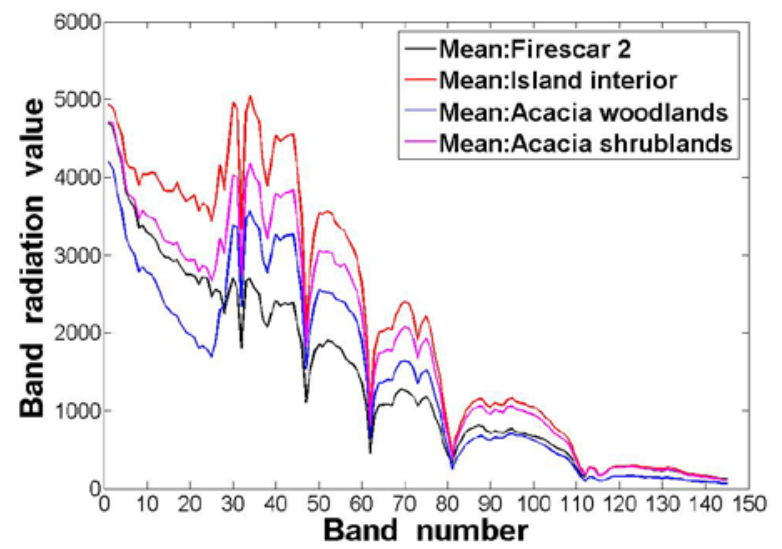

(c)Mean spectrum curve of $7^{\text {th }}$ to $10^{\text {th }}$ category.

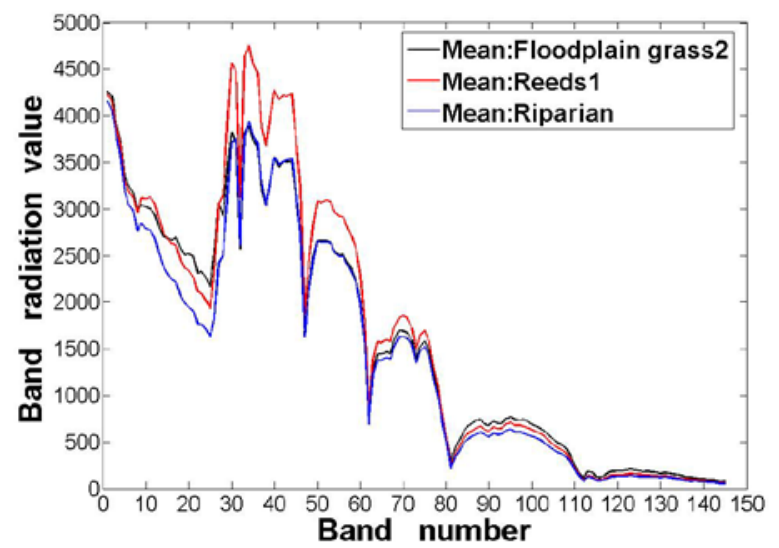

(b) Mean spectrum curve of $4^{\text {th }}$ to $6^{\text {th }}$ category.

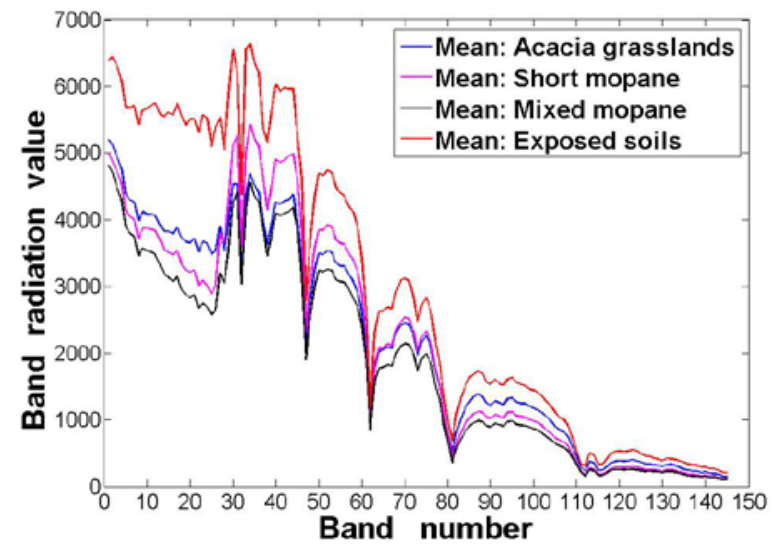

(d) Mean spectrum curve of $11^{\text {th }}$ to $14^{\text {th }}$ category.

Fig.2. Mean spectrum curve of $1^{\text {st }}$ to $14^{\text {th }}$ category

In figure 2, the spectral bands of 14 different kinds of materials have the similar structures. Thus, band division and sub-dictionary division based on band number of peaks and troughs of 14 categories are listed in table 1:

Table 1 Sub-dictionary Division of Botswana Hyperspectral Data

\begin{tabular}{lllllll}
\hline Sub-dictionary & D1 & D2 & D3 & D4 & D5 & D6 \\
\hline Band Region & $1-24$ & $25-47$ & $48-62$ & $63-81$ & $82-112$ & $113-145$ \\
\hline
\end{tabular}

\subsection{Experiment Result and Comparison}

In the experiment, firstly, 25\% data samples are selected randomly into the test data set, the rest of $75 \%$ data are used as the pool of training samples. Then four different training sets with different proportion of samples selected randomly from the training samples pool are investigated, the rates are $5 \%, 15 \%, 30 \%$ and 50\%. Each training sets with corresponding proportion are executed 5 times. Pixel values with respect to each band are normalized. The classification accuracy is the average of five experimental results. The classification method used for comparison is SR algorithm.

Table 2 compares the classification performances of SR algorithm with our improved algorithm at different sampling rates. 
Table 2. Classification AcCuracy of SR Algorithm AND Our Method

\begin{tabular}{lll}
\hline Training rate & SR algorithm & Our method \\
\hline $5 \%$ & 88.460 & 89.613 \\
$15 \%$ & 92.323 & 93.256 \\
$30 \%$ & 94.205 & 95.167 \\
$50 \%$ & 95.208 & 96.256 \\
$75 \%$ & 96.088 & 96.854 \\
\hline
\end{tabular}

Figure 3 shows the experimental results.

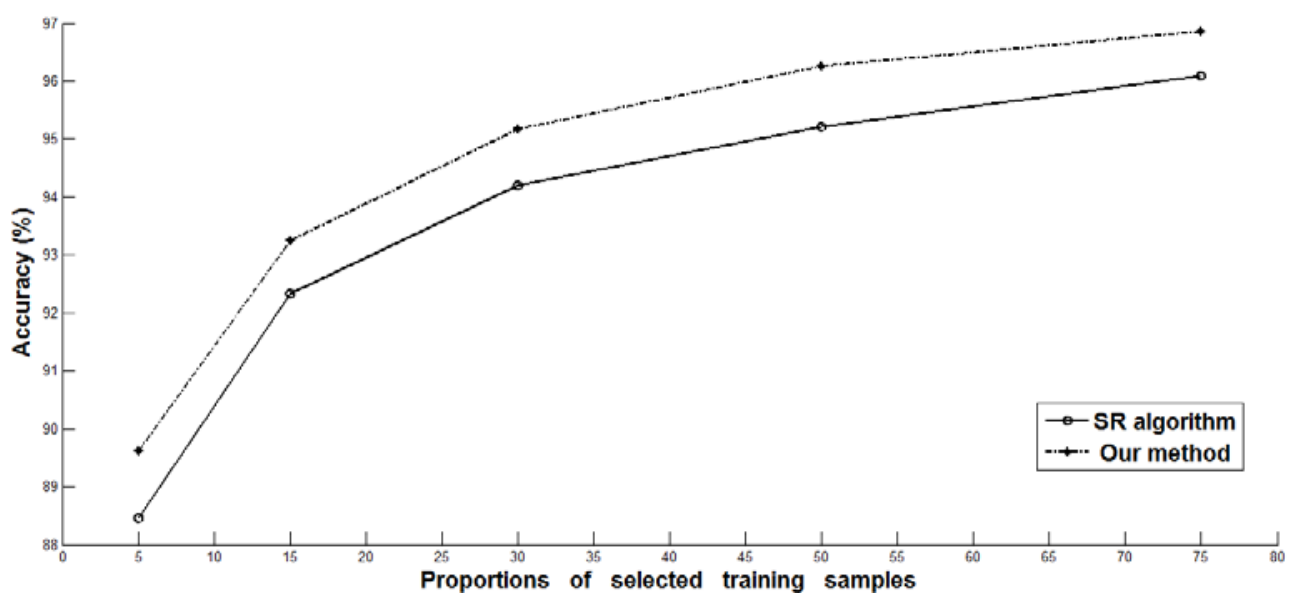

Fig.3. The experimental results

The overall results in table 2 and figure 3 for the Botswana data clearly demonstrate that the proposed method outperforms a standard SR algorithm in terms of accuracy, irrespective of the number of training samples.

\section{Conclusion}

In this paper, in order to improve discrimination performance of single dictionary based on SR algorithm, an improved classification method of sparse representation of hyperspectral images based on sub-dictionary division and weighted combination is proposed. The classification effectiveness of the proposed algorithm is verified by experiments.

\section{Acknowledgement}

In this paper, the research was sponsored by the National Natural Science Foundation of China (Project No. 61461002) and Innovation Projects for graduate students of Beifang University of Nationalities.

\section{References}

[1] S.G. Mallat and Z. F. Zhang "Matching pursuits with time-frequency dictionaries," IEEE transactions on Signal processing vol 41, No 12, Dec. 1993.

[2] Scott Shaobing Chen, David L. Donoho and Michael A. Saunders, “Atomic decomposition by basis pursuit,” 2001 Society for Industrial and Applied Mathematics, vol. 43, No. 1, pp. 129-159.

[3] David L. Donoho and X. M Huo, "Uncertainty principles and ideal atomic decomposition," IEEE transactions on Information Theory, vol. 47, No. 7, Nov 2001.

[4] J. A. Tropp. Greed is Good: Algorithmic results for sparse approximation [J]. Information Theory, IEEE Transactions on.50(10): 2231-2242(2004). 
[5] C. M. Zhang, Z. K. Yin, X. D. Chen and M. X. Xiao. Signal overcomplete representation and sparse decomposition based on redundant dictionaries[J]. Chinese Science Bulletin.50(23):26722677(2005).

[6] Adel Rahmoune, Pierre Vandergheynst, and Pascal Frossard, "The m-term pursuit for image representation and progressive compression,” IEEE International conference on Image Processing, 2005, 1:73-76.

[7] T. Guha and Rabab Ward, "A sparse reconstruction based algorithm for image and video classification," IEEE International Conference on Acoustics, Speech, and Signal Processing (ICASSP), pp.3601-3604, March. 2012.

[8] Alhussein Fawzi, Mike Davies and Pascal Frossard Dictionary Learning for fast classification based on soft-thresholding [J]. International Journal of Computer Vision . Feb 11, 2014.

[9] Qazi Sami ul Haq, L. M. Tao and F.C. Sun. “A fast and robust sparse approach for hyperspectral data classification using a few labelled samples,” IEEE Transactions on Geoscience and Remote Sensing, vol. 50, No. 6, Jun 2012 .

[10] X. T. Hao, C. M. Zhang, J. Bai, M. Dai and W. X. Bao. Classification modeling of multifeatured remote sensing images based on sparse representation. Computer Engineering and Networking. Springer International Publishing 2014 :577-584.

[11] G. L. Duan, L. Wei and N. Li. Adaptive weighted multiple sparse representation classification approach. Computer Engineering and Applications, 2014, 50 (8) : 173-177. 